University of Northern lowa

UNI ScholarWorks

Faculty Publications

Faculty Work

$10-21-2017$

\title{
Atomic disorder induced modification of magnetization in MnCrVAl
}

Juliana Herran

University of Northern lowa

Rishabh Dalal

University of Northern lowa

See next page for additional authors

Let us know how access to this document benefits you

Copyright @Herran, et al.

(c) (i)

This work is licensed under a Creative Commons Attribution 4.0 License.

Follow this and additional works at: https://scholarworks.uni.edu/phy_facpub

Part of the Physics Commons

\section{Recommended Citation}

Herran, Juliana; Dalal, Rishabh; Gray, Paul; Kharel, Parashu; and Lukashev, Pavel, "Atomic disorder induced modification of magnetization in MnCrVAl" (2017). Faculty Publications. 27.

https://scholarworks.uni.edu/phy_facpub/27

This Article is brought to you for free and open access by the Faculty Work at UNI ScholarWorks. It has been accepted for inclusion in Faculty Publications by an authorized administrator of UNI ScholarWorks. For more information, please contact scholarworks@uni.edu. 
Authors

Juliana Herran, Rishabh Dalal, Paul Gray, Parashu Kharel, and Pavel Lukashev 


\section{Atomic disorder induced modification of magnetization in MnCrVAl}

Cite as: J. Appl. Phys. 122, 153904 (2017); https://doi.org/10.1063/1.4998308

Submitted: 31 July 2017 . Accepted: 05 October 2017 . Published Online: 20 October 2017

Juliana Herran, Rishabh Dalal, Paul Gray (D), Parashu Kharel, and Pavel V. Lukashev
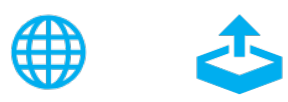

View Online

Export Citation

CrossMark

\section{ARTICLES YOU MAY BE INTERESTED IN}

Effect of disorder on the magnetic and electronic structure of a prospective spin-gapless semiconductor MnCrVAl

AIP Advances 7, 056402 (2017); https://doi.org/10.1063/1.4972797

Possible spin gapless semiconductor type behaviour in CoFeMnSi epitaxial thin films Applied Physics Letters 111, 152407 (2017); https://doi.org/10.1063/1.4996639

Magnetic tunnel junctions with an equiatomic quaternary CoFeMnSi Heusler alloy electrode Applied Physics Letters 112, 052403 (2018); https://doi.org/10.1063/1.5002763

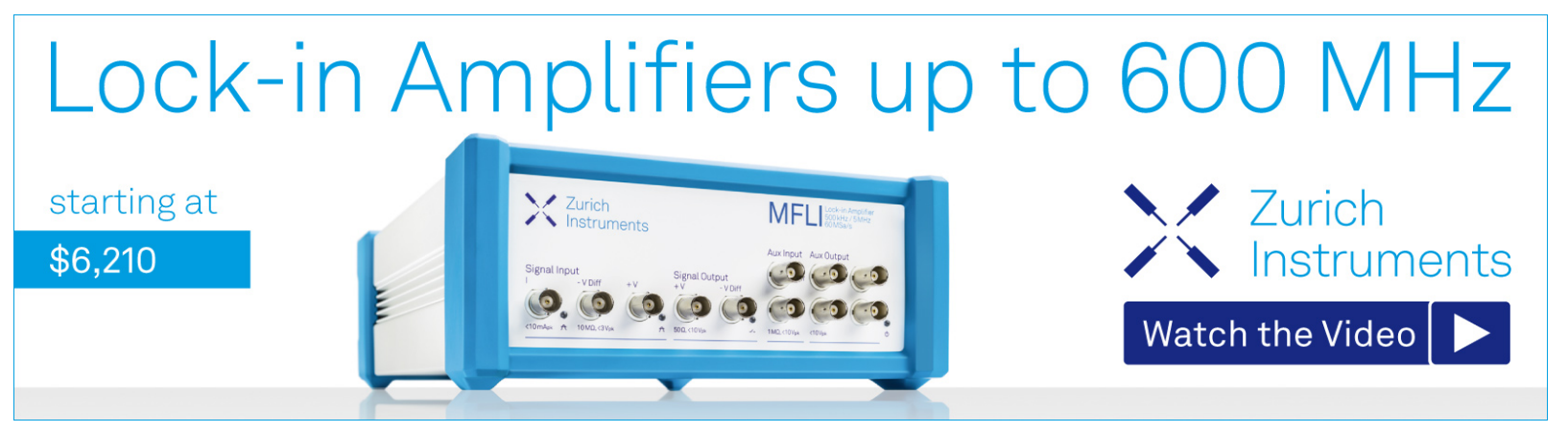




\title{
Atomic disorder induced modification of magnetization in MnCrVAl
}

\author{
Juliana Herran, ${ }^{1}$ Rishabh Dalal, ${ }^{2}$ Paul Gray, ${ }^{2}$ Parashu Kharel, ${ }^{3}$ and Pavel V. Lukashev ${ }^{4}$ \\ ${ }^{1}$ Department of Chemistry and Biochemistry, University of Northern Iowa, Cedar Falls, Iowa 50614, USA \\ ${ }^{2}$ Department of Computer Science, University of Northern Iowa, Cedar Falls, Iowa 50614, USA \\ ${ }^{3}$ Department of Physics, South Dakota State University, Brookings, South Dakota 57007, USA \\ ${ }^{4}$ Department of Physics, University of Northern Iowa, Cedar Falls, Iowa 50614, USA
}

(Received 31 July 2017; accepted 5 October 2017; published online 20 October 2017)

\begin{abstract}
We have investigated the physical mechanism behind magnetization reduction in a potential spingapless semiconducting compound MnCrVAl by analyzing various atomic disorder schemes. In particular, we show that depending on the degree of disorder, exchanging atomic positions between $\mathrm{Mn} / \mathrm{Cr}$ and $\mathrm{V} / \mathrm{Al}$ leads to reduced total magnetization due to either spin flip, or vanishing spin magnetic moments. The latter is attributed to the itinerant character of magnetism in $\mathrm{Cr}-$, $\mathrm{Mn}-$, and $\mathrm{V}$-containing Heusler alloys, and to the frustration of antiferromagnetic exchange interactions, and is accompanied by a tetragonal distortion, but such distortion alone (i.e., in a fully ordered crystal, with no atomic disorder) is not sufficient for a transition to zero magnetization. Besides, we demonstrate that in certain disordered structures the spin polarization of $\mathrm{MnCrVAl}$ significantly increases, reaching the half-metallic state. Our calculations indicate that exchange of atomic positions of $\mathrm{Mn}$ with $\mathrm{Cr}$, and $\mathrm{V}$ with $\mathrm{Al}$ has no significant effect on electronic and magnetic properties of MnCrVAl. We also show that antisite disorder does not result in significant reduction of magnetization. At the same time, some types of antisite disorder result in essentially $100 \%$ spin-polarized structures. These findings may contribute to understanding the role of atomic disorder on magnetic properties of materials with potential applications in spin-based electronics. Published by AIP Publishing.

https://doi.org/10.1063/1.4998308
\end{abstract}

\section{INTRODUCTION}

Research on magnetic materials for potential applications in spin-based electronics is one of the most active fields of current study in both academia and industry. ${ }^{1-6}$ In particular, magnetic materials which can produce highly spin polarized current (ideally, of only one spin orientation, in which case they are called half-metals) attracted enormous attention. Many compounds exhibiting high degree of transport spin polarization have been proposed. ${ }^{7-10}$ Although experimental confirmation of half-metallicity is rather challenging, it has been shown using spin-polarized positron-annihilation ${ }^{11,12}$ and infrared absorption ${ }^{13}$ that a semi-Heusler compound $\mathrm{NiMnSb}$ is a half-metal. Additionally, there are various mechanisms which could potentially alter the degree of transport spin polarization, such as mechanical strain, structural disorder, temperature, termination surface/interface in thin film multilayer geometry, etc. For example, it has been shown that segregation of atoms to the surface and emergence of surface states in thin film geometry produce detrimental effects on half-metallic properties. ${ }^{14-21}$

The main purpose of the current work is to investigate the effect of atomic disorder on the magnetic properties of $\mathrm{MnCrVAl}$, which has recently been investigated as a potential spin-gapless semiconducting (SGS) compound. ${ }^{22,23}$ Spin-gapless semiconductors are recently discovered materials which are characterized by a zero band gap in one spin channel and a finite band gap in the other channel. ${ }^{24-26}$ In SGS, not only electrons but also holes are $100 \%$ spin polarized and the spin-polarized electron conduction can be tuned between p-type and n-type by adjusting the gate voltage. SGS properties have been reported in oxides such as Co doped $\mathrm{PbPdO}_{2}$, and some Heusler alloys, such as $\mathrm{Mn}_{2} \mathrm{CoAl}$, $\mathrm{CoCrFeAl}$, etc. ${ }^{25,26}$ In our recent work, we demonstrated that in certain materials spin gapless semiconductivity could be induced by atomic substitution with the change in lattice parameters. $^{10}$

$\mathrm{MnCrVAl}$ belongs to the family of full Heusler compounds which crystallizes in a Y-structure (prototype LiMgPdSn) where $\mathrm{V}$ and $\mathrm{Al}$ occupy the Wyckoff's $4 \mathrm{a}$ and $4 \mathrm{~b}$ positions, respectively, while $\mathrm{Mn}$ and $\mathrm{Cr}$ atoms alternately occupy the $4 \mathrm{~b}$ and $4 \mathrm{~d}$ sites, which are all located in the middle of the bcc unit cells [see Fig. 1(a)]. In the completely ordered Y-structure, this material is theoretically predicted to be nearly SGS with net magnetic moment of $\approx 3.0 \mu_{\mathrm{B}} / \mathrm{f} . \mathrm{u}^{27}$ However, in our recent experimental work, we showed that MnCrVAl samples exhibit close to zero value of net magnetization. ${ }^{23}$ Here, we present detailed theoretical study of the physical mechanism responsible for the quenching of magnetic moment in this material. In particular, we show that certain states of experimentally observed atomic disorder result in vanishing spin magnetic moments.

The paper is organized as follows: In Sec. II, we present computational methods. In Sec. III, we outline our main results on electronic, magnetic, and structural properties of ordered and disordered $\mathrm{MnCrVAl}$, with various possible combinations of atomic disorder. In Sec. IV, we discuss the results and summarize the paper.

\section{METHODS}

We performed density functional theory (DFT) calculations of bulk Heusler compound, MnCrVAl using the projector augmented-wave method (PAW), ${ }^{28}$ implemented in the Vienna $a b$ initio simulation package $(\mathrm{VASP})^{29}$ within the 

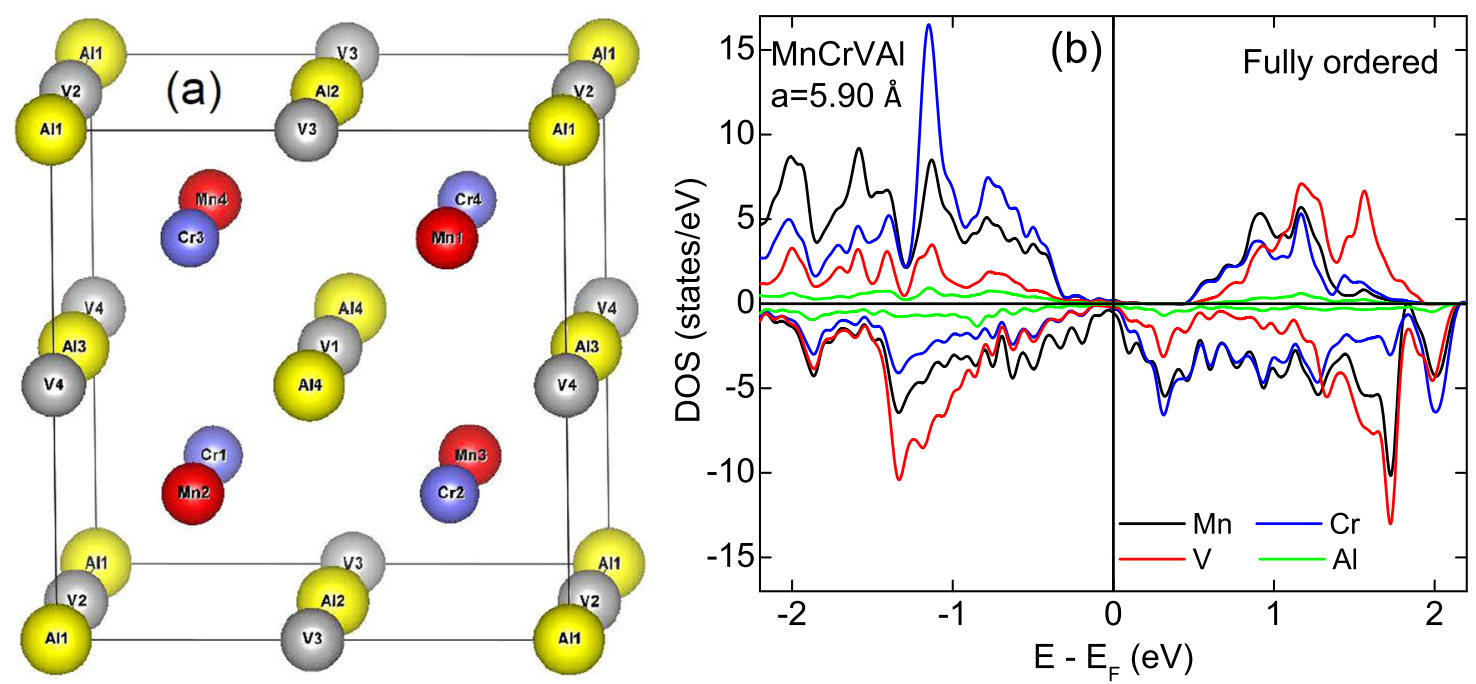

FIG. 1. (a) Crystal structure of a fully ordered MnCrVAl, and (b) atom- and spin-resolved densities of states of the bulk cubic MnCrVAl. Atomic contributions are color coded as indicated in the figure. Positive DOS corresponds to majority-, negative DOS to minority- spin states.

generalized-gradient approximation (GGA). ${ }^{30}$ The integration method, ${ }^{31}$ with a $0.05 \mathrm{eV}$ width of smearing was used, along with the plane-wave cut-off energy of $500 \mathrm{eV}$ and convergence criteria of $10^{-5} \mathrm{eV}$ for atomic relaxation, and $10^{-3} \mathrm{meV}$ for the total energy and electronic structure calculations. A $k$ point mesh of $12 \times 12 \times 12$ was used for the Brillouin-zone integration. The atomic arrangement of fully ordered 16-atom cubic unit cell used in our calculation is shown in Fig. 1(a). Here, $\mathrm{V}$ and $\mathrm{Al}$ occupy the Wyckoff's $4 \mathrm{a}$ and $4 \mathrm{~b}$ positions, respectively, while $\mathrm{Mn}$ and $\mathrm{Cr}$ atoms occupy the $4 \mathrm{~b}$ and $4 \mathrm{~d}$ sites, which are all located in the middle of the bcc unit cell. To simulate atomic disorder, we interchanged atomic positions of different elements, as explained in the text below. For all ground state calculations, the lattice parameters were fully optimized to obtain equilibrium structures. Periodic boundary condition was imposed in all calculations. Some of the results were obtained using the MedeA ${ }^{\circledR}$ software environment. ${ }^{32}$

\section{RESULTS AND DISCUSSION}

\section{A. Ideal structure}

Figure 1(a) shows the unit cell of the completely ordered MnCrVAl. The calculated optimal lattice constant is $5.90 \AA$. Figure 1(b) shows calculated densities of states (DOS) of this material at the ground state. Aside from a very small contribution from $\mathrm{Mn}$ and $\mathrm{Cr}$ atoms (which could be a computational artefact due to the energy width of smearing), the majorityspin DOS is essentially zero at the Fermi level with band gap of $\approx 0.5 \mathrm{eV}$. Further, the minority-spin DOS demonstrates a tendency towards gapless semiconducting behavior consistent with the previous report. ${ }^{27}$ As one can see from the Fig. 1(b), the maximum of the minority-spin valence band is mostly composed of Mn states, while the minimum of the unoccupied minority-spin states has comparable contribution from $\mathrm{Mn}, \mathrm{Cr}$, and $\mathrm{V}$.

The calculated magnetic structure of MnCrVAl in the ground state is ferrimagnetic, with net magnetic moment of $2.90 \mu_{\mathrm{B}} /$ f.u. The atomic contributions are $1.776 \mu_{\mathrm{B}}, 2.077 \mu_{\mathrm{B}}$, and $-0.943 \mu_{\mathrm{B}}$ per $\mathrm{Mn}, \mathrm{Cr}$, and $\mathrm{V}$ correspondingly (see
Table I). As mentioned earlier, this result is in contrast with our recently published experimental work where the arcmelted samples of MnCrVAl showed almost zero net magnetic moment. ${ }^{23}$ Our XRD measurements indicated a significant degree of atomic disorder in the samples. ${ }^{23}$ Here, we analyze how the magnetic, electronic, and structural properties of this material change as various disordered states are considered.

\section{B. Effect of swap disorder}

To investigate possible effects of atomic disorder on magnetic properties of $\mathrm{MnCrVAl}$, we constructed various cells of this alloy by interchanging atomic position in 16atom unit cell, as shown in Fig. 2. Here, Mn1-All notation indicates that the positions of Mn1 and Al1 atoms [see Fig. 1(a) for numbering of the atoms] have been interchanged, $\mathrm{Mn1}$, 2-Al1, 2 indicates that positions of Mn1 and Al1, and at the same time positions of $\mathrm{Mn} 2$ and $\mathrm{Al} 2$ are interchanged. All other disordered cells are labeled in the similar manner. Each of the structures was fully optimized. The calculated lattice parameters are presented in the Table I.

Table I summarizes the calculated magnetic moments for each of the structures shown in Fig. 2. One can see that atomic disorder in all the states except $\mathrm{Cr} 1-\mathrm{V} 1$ results in reduced magnetization (for $\mathrm{Mn} 1-\mathrm{V} 1$ this reduction is very small, but not negligible). Moreover, there are three instances of vanishing magnetization: Mn1,2-Al1,2, Mn1,2-V1,2, and $\mathrm{Cr} 1,2-\mathrm{A} 11,2$. In these three cases, the zero magnetization transition is accompanied by a cubic to tetragonal distortion, which is more pronounced in Mn1,2-V1,2. The last column of the Table I shows formation energies of the disordered structures, calculated as a total energy difference of ordered and disordered cell, i.e., less negative value indicates more stable structure. One can see that of the three disordered structures with zero magnetization, the highest formation energy (i.e., the least negative value) corresponds to $\mathrm{Cr} 1,2-$ Al1,2 disorder, while the second least negative value corresponds to Mn1,2-Al1,2 disorder. The formation energy of 
TABLE I. Calculated magnetic moments (in units of $\mu_{\mathrm{B}}$ ) and lattice constant (in $\AA$ ) of MnCrVAl in ordered and disordered states. In second column, "a/b/c" corresponds to the two in-plane $(a, b)$ and one out-of-plane $(c)$ lattice parameters. In columns 4, 6, 8, and 10, magnetic moments of 2nd, 3rd, and 4th Mn/Cr atoms [as shown in Fig. 1(a)] are listed. Except for Cr1, 2-V1, 2, the moments of 2nd, 3rd, and 4th atoms are the same for both Mn and Cr. The last two rows show calculated data of the fully ordered MnCrVAl, under compressive and tensile strain. The last column shows formation energies, calculated as $\mathrm{E}_{\text {total }}$ (ordered) - $\mathrm{E}_{\text {total }}$ (swap).

\begin{tabular}{|c|c|c|c|c|c|c|c|c|c|c|c|}
\hline & Lattice $(\mathrm{a} / \mathrm{b} / \mathrm{c})$ & Mn1 & $\mathrm{Mn} 2,3,4$ & $\mathrm{Cr} 1$ & $\mathrm{Cr} 2,3,4$ & V1 & $\mathrm{V} 2,3,4$ & Al1 & $\mathrm{Al} 2,3,4$ & Total & $E_{\text {formation }}$ \\
\hline Ordered & $5.90 / 5.90 / 5.90$ & 1.776 & 1.776 & 2.077 & 2.077 & -0.943 & -0.943 & -0.007 & -0.007 & 11.610 & \\
\hline Mn1-Al1 & $5.88 / 5.88 / 5.88$ & -1.741 & 1.632 & 1.189 & 1.716 & -1.007 & -0.864 & -0.018 & -0.012 & 5.840 & -0.514 \\
\hline Mn1-V1 & $5.91 / 5.91 / 5.91$ & -2.204 & 1.950 & 2.021 & 1.975 & 0.961 & -0.901 & -0.003 & 0.010 & 9.880 & -1.160 \\
\hline Mn1,2-Al1,2 & $5.84 / 5.77 / 5.84$ & 0.000 & 0.000 & 0.000 & 0.000 & 0.000 & 0.000 & 0.000 & 0.000 & 0.000 & -1.430 \\
\hline Mn1,2-V1,2 & $6.04 / 5.73 / 5.73$ & 0.000 & 0.000 & 0.000 & 0.000 & 0.000 & 0.000 & 0.000 & 0.000 & 0.000 & -2.910 \\
\hline Cr1-Al1 & $5.87 / 5.875 .87$ & 1.122 & 1.694 & -1.359 & 1.600 & -1.077 & -0.837 & -0.030 & -0.013 & 5.99 & -0.236 \\
\hline Cr1-V1 & $5.90 / 5.87 / 5.87$ & 1.911 & 1.836 & -1.303 & 2.105 & 1.558 & -0.891 & -0.005 & 0.008 & 11.34 & -0.689 \\
\hline $\mathrm{Cr} 1,2-\mathrm{A} 11,2$ & $5.78 / 5.78 / 5.91$ & 0.000 & 0.000 & 0.000 & 0.000 & 0.000 & 0.000 & 0.000 & 0.000 & 0.000 & -1.001 \\
\hline \multirow[t]{3}{*}{$\mathrm{Cr} 1,2-\mathrm{V} 1,2$} & $5.91 / 5.90 / 5.91$ & -0.522 & 1.558 & -0.528 & -0.528 & -0.357 & -0.523 & 0.005 & 0.010 & 1.88 & -2.050 \\
\hline & & & 1.558 & & 1.691 & & -0.329 & & 0.010 & & \\
\hline & & & -1.530 & & 1.691 & & -0.329 & & 0.005 & & \\
\hline Compressive & $5.8 / 5.8 / 5.966$ & 1.720 & 1.720 & 2.051 & 2.051 & -0.880 & -0.880 & -0.003 & -0.003 & 11.55 & \\
\hline Tensile & $6.0 / 6.0 / 5.817$ & 1.849 & 1.849 & 2.105 & 2.105 & -1.022 & -1.022 & -0.013 & -0.013 & 11.68 & \\
\hline
\end{tabular}

Mn1,2-V1,2 is significantly higher ( $\mathrm{E}_{\text {formation }}$ is more negative). In all considered cases, the ordered structure corresponds to the lowest energy state. As discussed in Ref. 23, the XRD measurements indicate a significant degree of atomic disorder in the samples, which could be caused by various reasons, e.g., preparation methods. We therefore argue that experimentally observed zero magnetization in $\mathrm{MnCrVAl}$ could result from atomic swap disorder, possibly of Cr1,2-Al1,2 and Mn1,2-Al1,2 type.

Next, we look at the electronic structure of disordered cells. Figures 3 and 4 show atom- and spin- resolved densities of states of the cells with Mn-type and Cr-type (interchange of $\mathrm{Mn}$ atoms and $\mathrm{Cr}$ atoms correspondingly) atomic disorder. As shown in Figs. 3(c) and 3(d) and Fig. 4(c), Mn1,2-Al1,2, Mn1,2-V1,2, and Cr1,2-Al1,2 disordered cells exhibit non-spin-polarized electronic structure, corresponding to vanishing spin magnetic moments. In particular, as a result of atomic disorder in these structures, new states emerge near Fermi level, mostly of $\mathrm{Mn}, \mathrm{Cr}$, and $\mathrm{V}$ character (with comparable contribution from all three elements).
These states essentially cancel out the spin polarization. The other five disordered cells demonstrate various degrees of non-zero spin-polarization, which is especially pronounced for Cr1-Al1 disorder, which exhibits a nearly half-metallic electronic structure.

All three non-magnetic disordered cells exhibit tetragonal distortion; see the second column of the Table I. It is important to understand if the tetragonal distortion without atomic disorder can induce the transition to zero magnetization. In order to find an answer to this question, we performed calculations for a fully ordered MnCrVAl, under both compressive and tensile strain. For the former, we set the in-plane lattice constant to $5.80 \AA$, while for the latter we set it to $6.00 \AA$ (i.e., $\pm 1.7 \%$ off the cubic ground state value). In both cases, the out-of-plane lattice parameter was fully relaxed, with the optimal values of $5.966 \AA$ for compressive, and $5.817 \AA$ for tensile strain. In both cases, magnetic and electronic structures are not significantly different from the corresponding structures of the fully ordered cubic cell, i.e., the spin magnetic moments

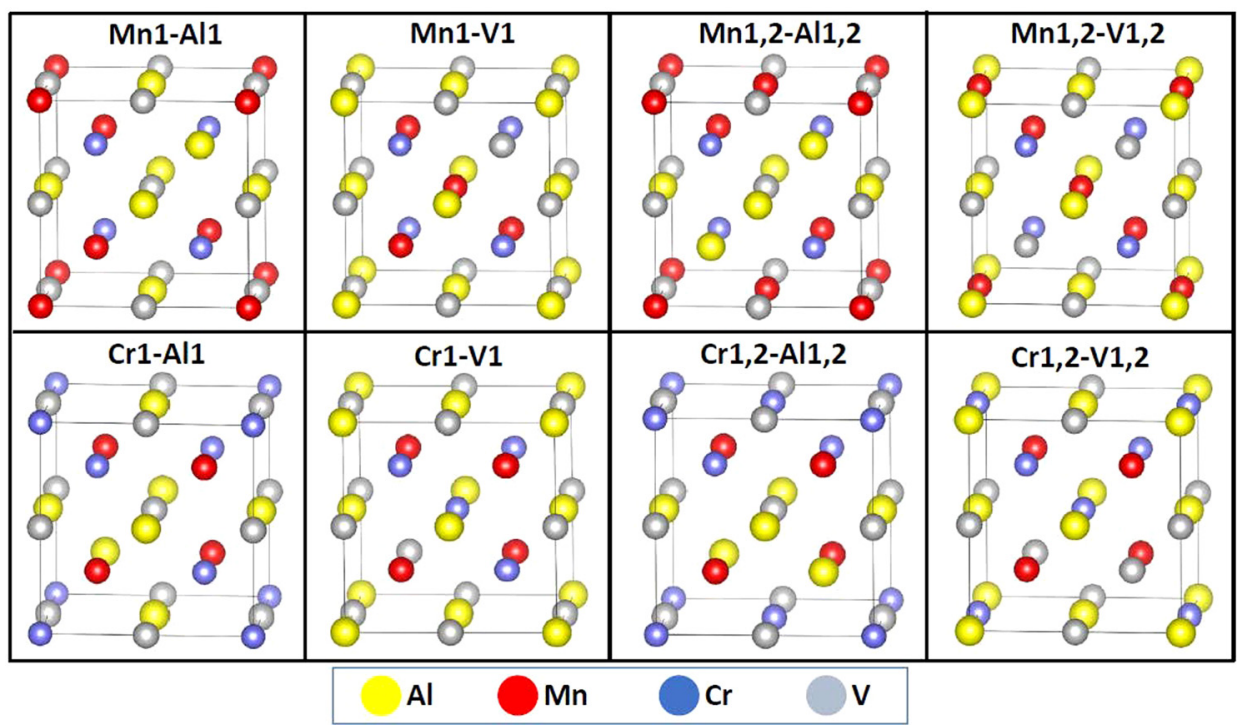

FIG. 2. Crystal structures of disordered MnCrVAl. 


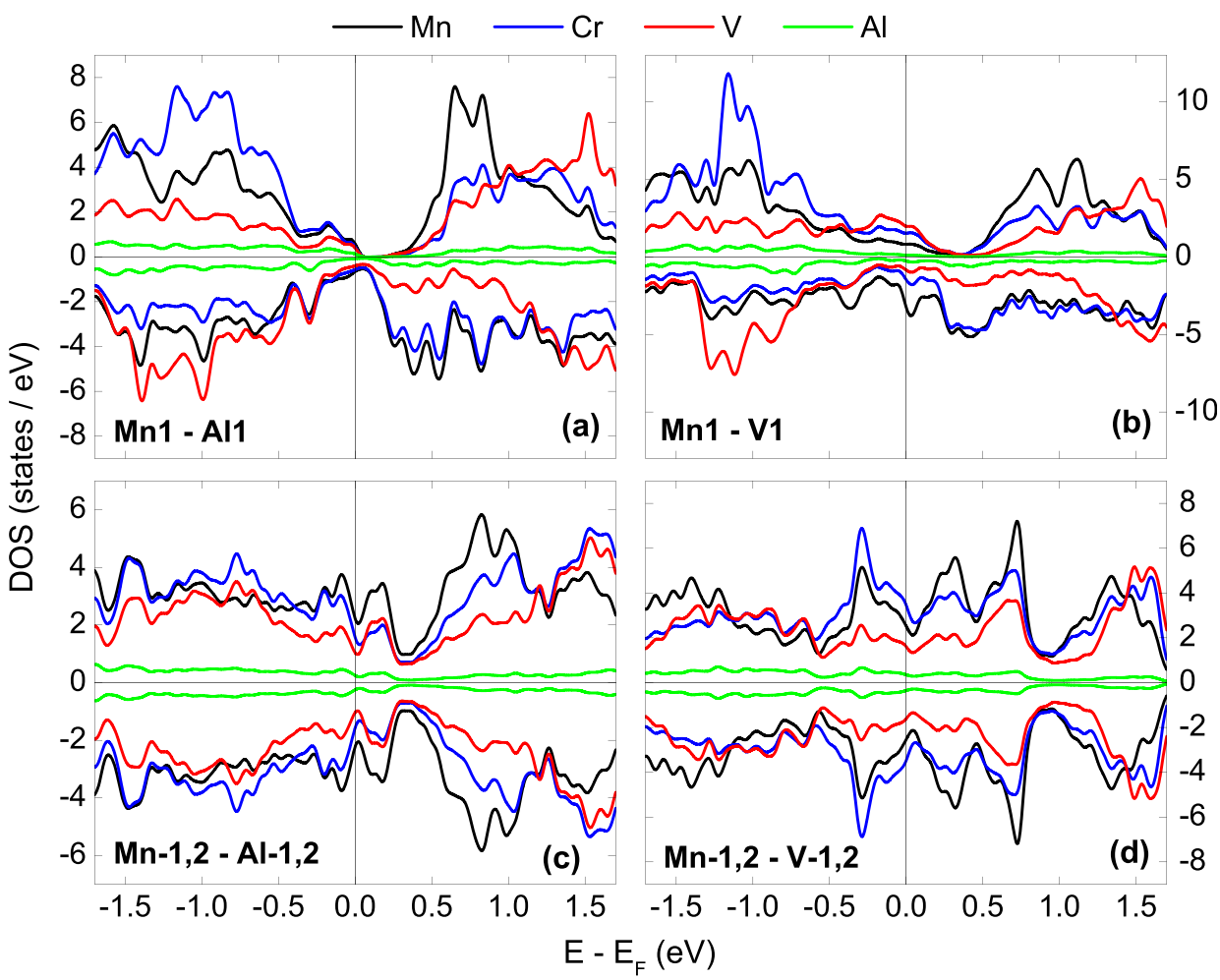

FIG. 3. Calculated densities of states of cells with Mn-type disorder. Atomic contributions are color coded as indicated in the figure. Positive DOS corresponds to majority-, negative DOS to minority- spin states.

are not vanishing. The last two rows in Table I show the calculated magnetic moments of fully ordered cells under compressive and tensile strain, while Fig. 5 shows the calculated densities of states of these two structures. Comparison of Figs. 1(b) and 5(a), 5(b) shows that the electronic structure of MnCrVAl is not very sensitive to the external strain, as both total and atom-projected DOS exhibit similar features under zero and positive/negative compression.
To better illustrate the nature of the atomic disorder induced magnetization modification in $\mathrm{MnCrVAl}$, we plot average planar magnetization density in the unit cell of fully ordered and disordered structures (see Fig. 6). In Fig. 6, the two positive magnetization peaks correspond to $z=0.25$ and $z=0.75$ (direct coordinates). This corresponds to two planes which contain $\mathrm{Mn}$ and $\mathrm{Cr}$ atoms in the fully ordered unit cell [see Fig. 1(a)]. The negative peaks correspond to $z=0.0$, $z=0.5$, and $z=1.0$, i.e., planes containing $\mathrm{Al}$ and $\mathrm{V}$ atoms in

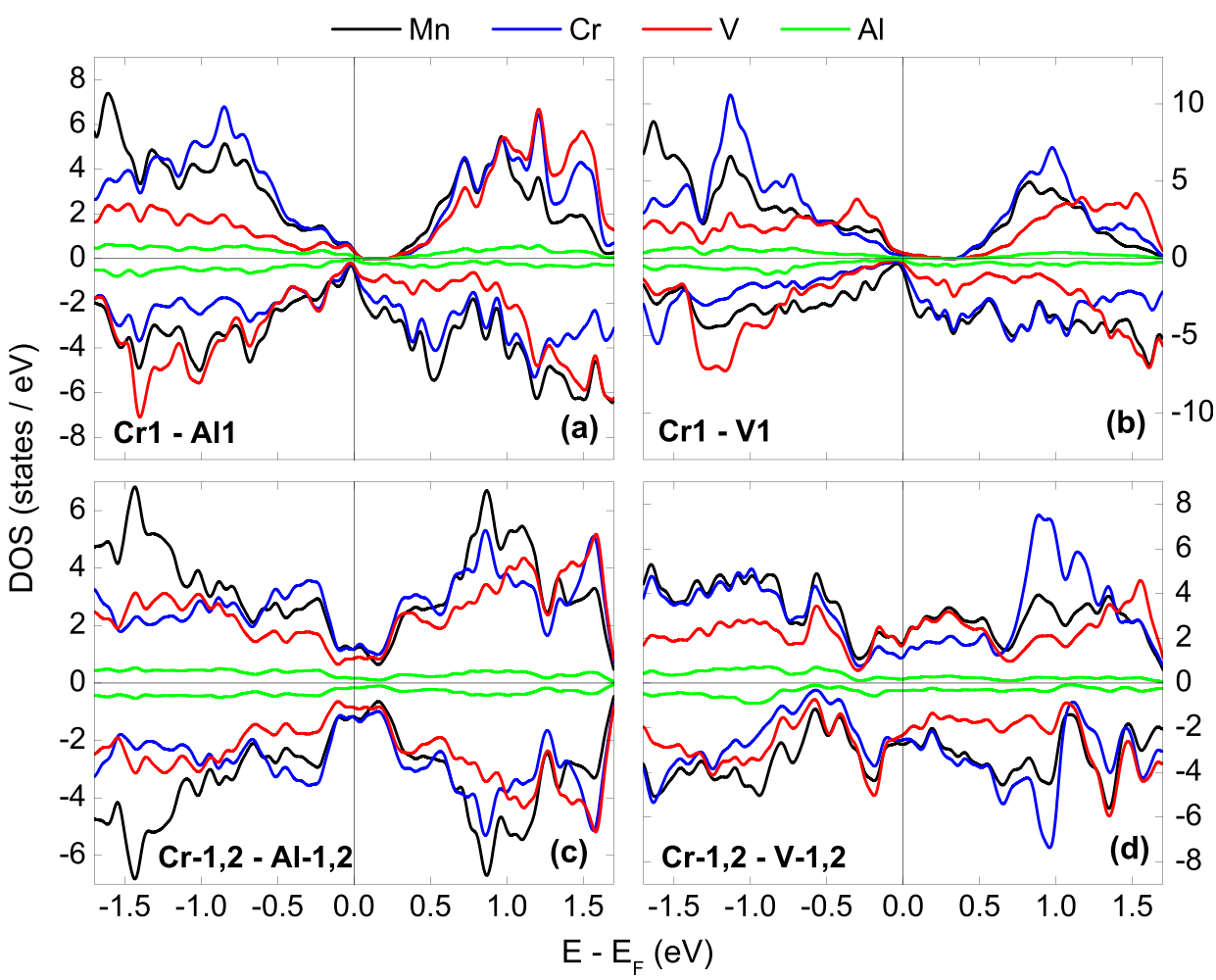

FIG. 4. Calculated densities of states of cells with Cr-type disorder. Atomic contributions are color code as indicated on the figure. Positive DOS corresponds to majority-, negative DOS to minority- spin states. 

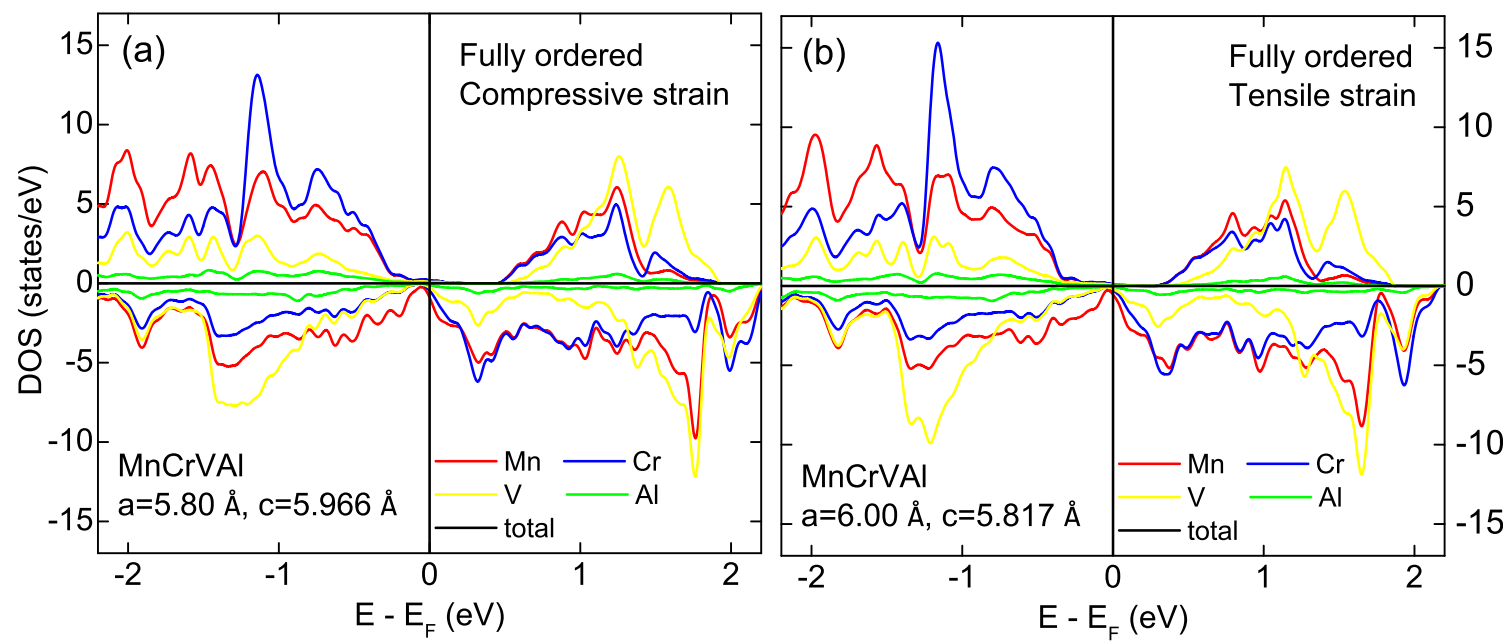

FIG. 5. Calculated densities of states of fully ordered MnCrVAl, under compressive (a) and tensile (b) strain. Atomic contributions are color code as indicated in the figure. Positive DOS corresponds to majority-, negative DOS to minority- spin states.

the ordered cell, and result from the spin-down orientation of the magnetic moment of vanadium. (Note that Fig. 6 shows distance in $z$-direction in Cartesian (not direct) coordinates.) As one can see, $\mathrm{Cr} 1-\mathrm{V} 1$ and Mn1-V1 atomic disorders do not significantly change magnetization density in the cell [compare black and red lines in Figs. 6(a) and 6(b)]. At the same time, Cr1-Al1 and Mn1-Al1 disorders result in nearly identical modification of the magnetization density profile, which reduces approximately twice the total magnetization. Except $\mathrm{Cr} 1,2-\mathrm{V} 1,2$, all disordered cells with interchange of two atoms exhibit zero magnetization density. Cr1,2-V1,2 disorder also results in a strong suppression of the total magnetization density [gray line in Fig. 6(a)], which, however, is not vanishing. Overall, magnetization suppression is much stronger when atomic positions of $\mathrm{Cr} / \mathrm{Mn}$ are interchanged with $\mathrm{Al}$ than with $\mathrm{V}$. This is likely due to the strong hybridization between non-spin polarized states of $\mathrm{Al}$ with spinpolarized states of $\mathrm{Cr} / \mathrm{Mn}$ when positions of $\mathrm{Al}$ and $\mathrm{Cr} / \mathrm{Mn}$ are interchanged, which results in magnetization quenching. On the other hand, when $\mathrm{Cr} / \mathrm{Mn}$ positions are interchanged with $\mathrm{V}$, the magnetization reduction is less pronounced, since both $\mathrm{V}$ and $\mathrm{Cr} / \mathrm{Mn}$ states are spin-polarized and their increased overlap has a smaller effect on magnetization profile.

Finally, we also performed atomic disorder calculations with the exchange of atomic positions of $\mathrm{Mn}$ with $\mathrm{Cr}$, and $\mathrm{V}$ with $\mathrm{Al}$. This type of disorder has no significant effect on the electronic and magnetic properties of $\mathrm{MnCrVAl}$, i.e., both magnetic and electronic structures show no noticeable deviation from that of the fully ordered cell.

\section{Effect of antisite disorder}

Recently, Feng et al. demonstrated that along with the swap disorder, antisite disorder may also have an important effect on the electronic and magnetic properties of some Heusler alloys. ${ }^{33}$ To investigate the effect of the antisite disorder on MnCrVAl, we constructed supercells of 32 atoms. In ordered structure, there are 8 atoms of each element. We consider all 12 possible types of antisite disorder: $\mathrm{Al}(\mathrm{Cr})$, $\mathrm{Al}(\mathrm{Mn}), \mathrm{Al}(\mathrm{V}), \mathrm{Cr}(\mathrm{Al}), \mathrm{Cr}(\mathrm{Mn}), \mathrm{Cr}(\mathrm{V}), \mathrm{Mn}(\mathrm{Al}), \mathrm{Mn}(\mathrm{Cr})$, $\mathrm{Mn}(\mathrm{V}), \mathrm{V}(\mathrm{Al}), \mathrm{V}(\mathrm{Cr})$, and $\mathrm{V}(\mathrm{Mn})$. In each of these 12 structures, one of the atoms of the element shown in parentheses is replaced with an atom preceding parenthesis [e.g., one
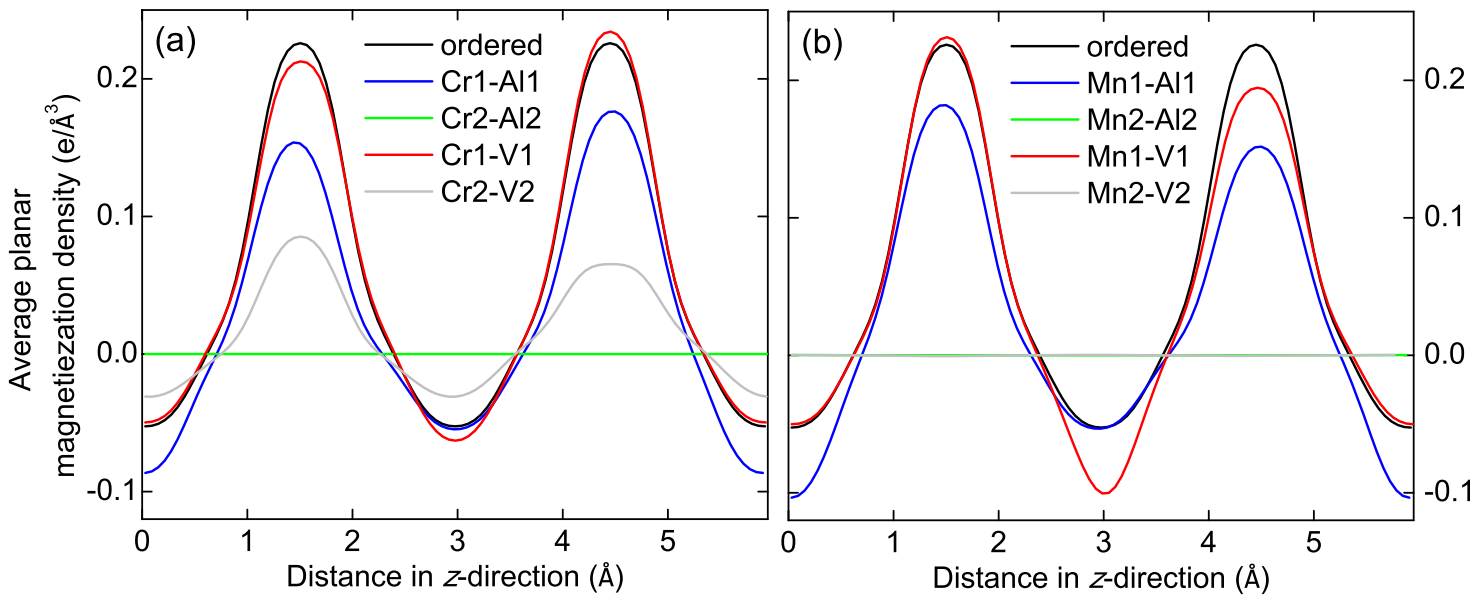

FIG. 6. Average planar magnetization density in Cr-type (a) and Mn-type (b) disordered MnCrVAl. To save space in figure legends, we replaces Cr1,2-Al1,2 (and similar) notations with $\mathrm{Cr} 2-\mathrm{Al} 2$, etc. 
TABLE II. Calculated magnetic moments (in units of $\mu_{\mathrm{B}} / 16$-atom cell) of 12 structures with antisite disorder.

\begin{tabular}{lcccccccccccccc}
\hline \hline & $\mathrm{Al}(\mathrm{Cr})$ & $\mathrm{Al}(\mathrm{Mn})$ & $\mathrm{Al}(\mathrm{V})$ & $\mathrm{Cr}(\mathrm{Al})$ & $\mathrm{Cr}(\mathrm{Mn})$ & $\mathrm{Cr}(\mathrm{V})$ & $\mathrm{Mn}(\mathrm{Al})$ & $\mathrm{Mn}(\mathrm{Cr})$ & $\mathrm{Mn}(\mathrm{V})$ & $\mathrm{V}(\mathrm{Al})$ & $\mathrm{V}(\mathrm{Cr})$ & $\mathrm{V}(\mathrm{Mn})$ \\
\hline $\mathbf{M}$ & 10.45 & 9.98 & 10.79 & 10.32 & 11.47 & 11.74 & 10.48 & 12.09 & 10.97 & 12.82 & 11.47 & 12.07 \\
\hline \hline
\end{tabular}

atom of $\mathrm{Cr}$ is replaced with $\mathrm{Al}$ in $\mathrm{Al}(\mathrm{Cr})]$. The calculated magnetic moments are summarized in Table II. One can see that all antisite disorder structures exhibit large magnetization. We therefore conclude that experimentally observed quenching of magnetization cannot result from antisite disorder. Calculated densities of states of all 12 structures with antisite disorder are shown in Fig. 7. One can see that while all of these disorders result in additional minority-spin states around Fermi level, yet some of these structures $[\mathrm{Al}(\mathrm{Cr})$, $\mathrm{Al}(\mathrm{Mn}), \mathrm{Cr}(\mathrm{Mn})$, and $\mathrm{V}(\mathrm{Cr})]$ exhibit nearly perfect halfmetallicity and therefore could have practical applicability.

\section{DISCUSSION AND CONCLUSIONS}

Our calculations indicate that atomic disorder induced decrease in the total spin magnetic moment in $\mathrm{MnCrVAl}$ is due to two physical mechanisms: induced antiferromagnetic exchange interaction (spin flip) and vanishing of the atomic spin moments. The latter is likely responsible for the experimentally observed zero magnetization in this alloy. ${ }^{23}$ The atomic disorder induced antiferromagnetic coupling is $\mathrm{Mn} /$ $\mathrm{Cr} / \mathrm{V}$ atoms could be due to the increased overlap of the corresponding $d$ orbitals, which results from reduced interatomic distances. ${ }^{34,35}$ At the same time, vanishing of the atomic spin moments in Mn1,2-Al1,2, Mn1,2-V1,2, and Cr1,2-Al1,2 disordered cells is probably due to a subtler mechanism. In particular, a similar reduction of the spin magnetic moment in another Heusler material, $\mathrm{Cr}_{3} \mathrm{Se}$ has been reported by Galanakis et al., and was attributed to the metamagnetic behavior of this material, i.e., coexistence of itinerant and localized spin magnetic moments. ${ }^{36}$ It is plausible to suggest that a similar mechanism may be responsible for the vanishing spin moments in disordered MnCrVAl. First, Cr-, Mn-, and V-containing Heusler alloys are likely to exhibit itinerant magnetism, and second, the disorder induced cell compression is comparatively small (see Table I), and is

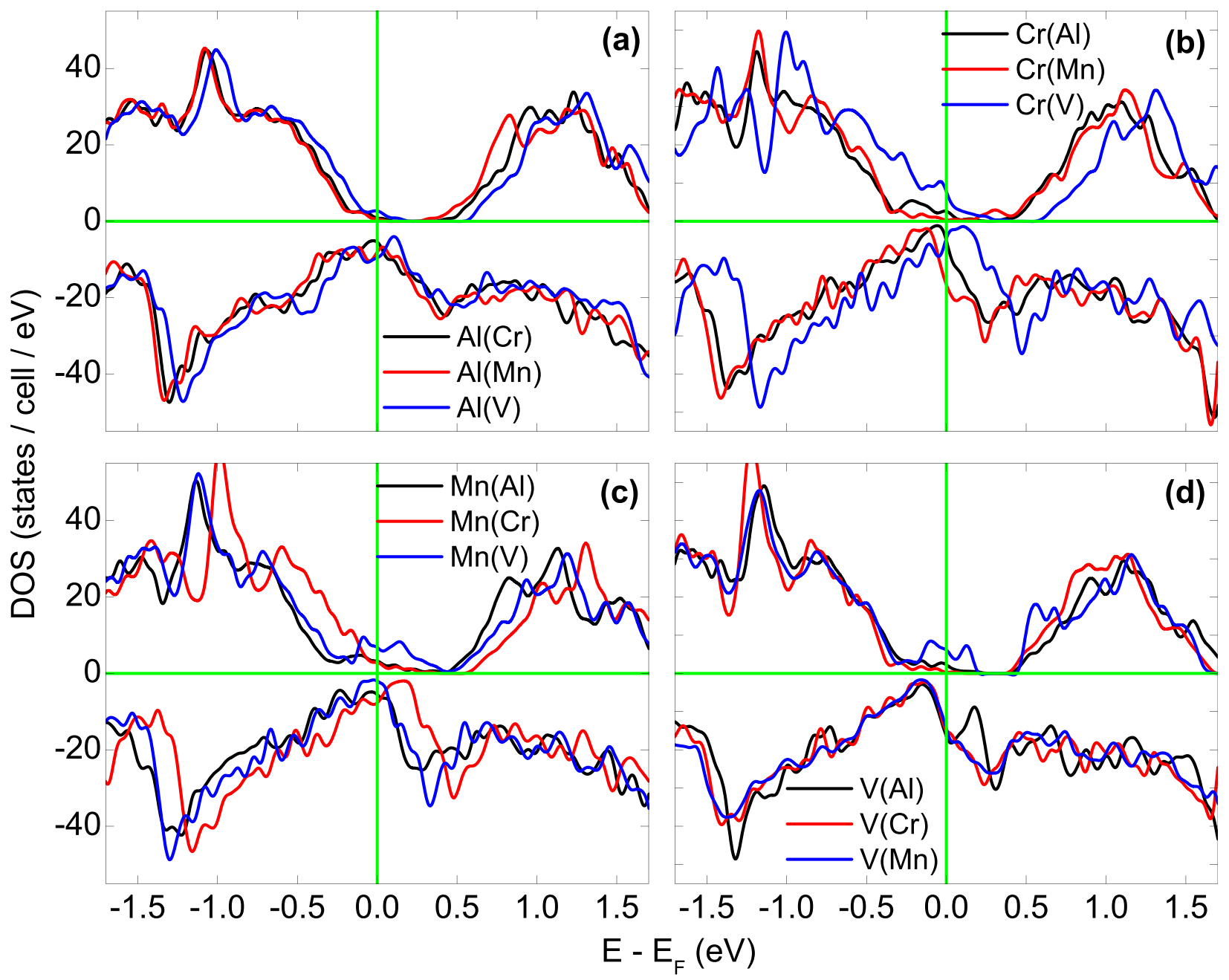

FIG. 7. Calculated total densities of states of the structures with antisite disorder. Positive DOS corresponds to majority-, negative DOS to minority- spin states. 
very unlikely to result in disappearance of the spin moments, if these moments are strongly localized (itinerant magnetic moments are reduced significantly faster). ${ }^{37}$ See Refs. 36 and 37 for further discussion of metamagnetism in Heusler alloys.

Results presented in this work are in overall agreement with our recent experimental study. ${ }^{23}$ In particular, in our experimental work, we used XRD measurements to show that $\mathrm{MnCrVAl}$ samples that exhibit zero magnetization have very strong A2-type disorder, which corresponds to the Mn$\mathrm{Al}$ and $\mathrm{Cr}-\mathrm{Al}$ atomic swap. At the same time, our XRD measurements did not indicate any B2-type atomic disorder, which corresponds to the $\mathrm{V}-\mathrm{Al}$ atomic swap. This is consistent with our first-principles results, which indicate that while A2-type disordered cells exhibit reduced magnetization, B2-type disorder does not significantly affect magnetic structure of the cell.

In conclusion, we showed that atomic swap disorder in $\mathrm{MnCrVAl}$ mostly results in either reduced total magnetization due to spin flip or in vanishing spin magnetic moments. The latter is accompanied by a tetragonal distortion, while all disordered cells with non-zero magnetization are cubic. Further, we also showed that tetragonal distortion alone (with no atomic disorder) is not sufficient for a suppression of magnetization. Besides, we demonstrated that although atomic disorder typically results in reduced spin-polarization of MnCrVAl, yet in certain disordered structures (Cr1-Al1) the spin polarization is very high, almost reaching the halfmetallic state. Our calculations indicate that exchange of atomic positions of $\mathrm{Mn}$ with $\mathrm{Cr}$, and $\mathrm{V}$ with $\mathrm{Al}$ has no significant effect on electronic and magnetic properties of MnCrVAl. We also showed that while antisite disorder does not result in significant reduction of magnetization, yet some types of this disorder may result in nearly $100 \%$ spinpolarized structures. Finally, although not considered in this work, but frustrated exchange interactions in some forms of A2-type atomic disorder may result in non-collinear magnetic structure, potentially exhibiting magnetic vortices (skyrmions). Reliable study of this effect using standard DFT techniques would be computationally very demanding, but may be an interesting subject of future investigation. Another potentially interesting topic of future study is magnetic domain structure of MnCrVAl and, in particular, its response to atomic disorder. Such an investigation, however, would also require beyond standard DFT methods. Overall, we hope that our findings are important for understanding the effect of atomic disorder on magnetic properties of materials with potential applications in spintronics.

\section{ACKNOWLEDGMENTS}

This research was supported by the Pre-Tenure Grant from the Office of the Provost and Executive Vice President for Academic Affairs, UNI, as well as by the UNI Faculty Summer Fellowship. Computations were performed at the Department of Physics computing facilities, UNI. The work at South Dakota State University (SDSU) was supported by Research/Scholarship Support Fund, SDSU.
${ }^{1}$ E. E. Y. Tsymbal and I. Žutić, Handbook of Spin Transport and Magnetism (CRC Press, Boca Raton, FL, 2011), p. 808.

${ }^{2}$ R. A. de Groot, F. M. Mueller, P. G. v. Engen, and K. H. J. Buschow, Phys. Rev. Lett. 50(25), 2024-2027 (1983).

${ }^{3}$ I. H. A. I. Galanakis, in Springer Series in Materials Science, edited by C. Felser and A. Hirohata (Springer International Publishing, Switzerland, 2016), p. 222.

${ }^{4}$ E. Şaşıŏglu, L. M. Sandratskii, P. Bruno, and I. Galanakis, Phys. Rev. B 72(18), 184415 (2005).

${ }^{5}$ G. H. F. B. Balke, J. Winterlik, and C. Felser, Appl. Phys. Lett. 90, 152504 (2007).

${ }^{6}$ I. Tutic, J. Herran, B. Staten, P. Gray, T. Paudel, A. Sokolov, E. Tsymbal, and P. Lukashev, J. Phys.: Condens. Matter 29, 075801 (2017).

${ }^{7}$ H. Kurt, K. Rode, M. Venkatesan, P. Stamenov, and J. Coey, Phys. Status Solidi B 248, 2338 (2011).

${ }^{8}$ J. Winterlik, S. Chadov, A. Gupta, V. Alijani, T. Gasi, K. Filsinger, B. Balke, G. Fecher, C. Jenkins, F. Casper, J. Kübler, G.-D. Liu, L. Gao, S. Parkin, and C. Felser, Adv. Mater. 24, 6283 (2012).

${ }^{9}$ A. Nelson, P. Kharel, Y. Huh, R. Fuglsby, J. Guenther, W. Zhang, B. Staten, P. Lukashev, S. Valloppilly, and D. Sellmyer, J. Appl. Phys. 117, 153906 (2015).

${ }^{10}$ P. Lukashev, P. Kharel, S. Gilbert, B. Staten, N. Hurley, R. Fuglsby, Y. Huh, S. Valloppilly, W. Zhang, K. Yang, R. Skomski, and D. Sellmyer, Appl. Phys. Lett. 108, 141901 (2016).

${ }^{11}$ K. Hanssen and P. Mijnarends, Phys. Rev. B 34, 5009 (1986).

${ }^{12}$ K. Hanssen, P. Mijnarends, L. Rabou, and K. Buschow, Phys. Rev. B 42, 1533 (1990).

${ }^{13}$ W. van Roy, M. Wojcik, E. Jdryka, S. Nadolski, D. Jalabert, B. Brijs, G. Borghs, and J. De Boeck, Appl. Phys. Lett. 83, 4214 (2003).

${ }^{14}$ F. Mancoff, B. Clemens, E. Singley, and D. Basov, Phys. Rev. B 60, R12565(R) (1999).

${ }^{15}$ W. Zhu, B. Sinkovic, E. Vescovo, C. Tanaka, and J. Moodera, Phys. Rev. B 64, 060403(R) (2001).

${ }^{16}$ G. Bona, F. Meier, M. Taborelli, E. Bucher, and P. Schmidt, Solid State Commun. 56, 391 (1985).

${ }^{17}$ S. Clowes, Y. Mioyoshi, Y. Bugoslavsky, W. Branford, C. Grigorescu, S. Manea, O. Monnereau, and L. Cohen, Phys. Rev. B 69, 214425 (2004).

${ }^{18}$ A. Caruso, C. Borca, D. Ristoiu, J. Nozieres, and P. Dowben, Surf. Sci. 525, L109 (2003).

${ }^{19}$ D. Ristoiu, J. Nozières, C. Borca, T. Komesu, H.-K. Jeong, and P. Dowben, Europhys. Lett. 49, 624 (2000).

${ }^{20}$ D. Ristoiu, D. J. Nozières, C. Borca, B. Borca, and P. Dowben, Appl. Phys. Lett. 76, 2349 (2000).

${ }^{21}$ P. Lukashev, J. Burton, A. Smogunov, J. Velev, and E. Tsymbal, Phys. Rev. B 88, 134430 (2013).

${ }^{22}$ X. L. Wang, Phys. Rev. Lett. 100(15), 156404 (2008).

${ }^{23}$ P. Kharel, J. Herran, P. Lukashev, Y. Jin, J. Waybright, S. Gilbert, B. Staten, P. Gray, S. Valloppilly, Y. Huh, and D. J. Sellmyer, AIP Adv. 7, 056402 (2017).

${ }^{24}$ H. Y. Jia, X. F. Dai, L. Y. Wang, R. Liu, X. T. Wang, P. P. Li, Y. T. Cui, and G. D. Liu, AIP Adv. 4, 047113 (2014).

${ }^{25}$ P. Kharel, W. Zhang, R. Skomski, S. Valloppilly, Y. Huh, R. Fuglsby, S. Gilbert, and D. J. Sellmyer, J. Phys. D: Appl. Phys. 48, 245002 (2015).

${ }^{26}$ S. Ouardi, G. H. Fecher, and C. Felser, Phys. Rev. Lett. 110, 100401 (2013).

${ }^{27}$ K. Özdoğan, E. Şaşığlu, and I. Galanakis, J. Appl. Phys. 113(19), 193903 (2013).

${ }^{28}$ P. E. Blöchl, Phys. Rev. B 50(24), 17953-17979 (1994).

${ }^{29}$ G. Kresse and D. Joubert, Phys. Rev. B 59(3), 1758-1775 (1999).

${ }^{30}$ J. P. Perdew, K. Burke, and M. Ernzerhof, Phys. Rev. Lett. 77(18), 3865-3868 (1996).

${ }^{31}$ M. Methfessel and A. T. Paxton, Phys. Rev. B 40(6), 3616-3621 (1989).

${ }^{32}{\text { Mede } A^{\circledR}}^{(}$Version 2.19. MedeA ${ }^{\circledR}$ is a registered trademark of Materials Design, Inc. Angel Fire, New Mexico, USA.

${ }^{33}$ Y. Feng, H. Chen, H. Yuan, Y. Zhou, and X. Chen, J. Magn. Magn. Mater. 378, 7 (2015).

${ }^{34}$ E. Şaşıŏlu, L. M. Sandratskii, and P. Bruno, J. Phys.: Condens. Matter 17, 995 (2005).

${ }^{35}$ I. Galanakis and E. Şaşıŏlu, Appl. Phys. Lett. 99, 052509 (2011).

${ }^{36}$ I. Galanakis, K. Özdoğan, and E. Şaşıoğlu, Phys. Rev. B 86, 134427 (2012).

${ }^{37}$ I. Galanakis, Ș. Tırpancı, K. Özdoğan, and E. Şaşığlu, Phys. Rev. B 94, 064401 (2016). 